\title{
Electronic Structure, NMR, Spin-spin Coupling and Noncovalent Interactions in Aromatic Amino Acid based Ionic Liquids.
}

\author{
Soniya S. Rao and Shridhar P. Gejji* \\ Department of Chemistry, Savitribai Phule Pune University, Pune 411 007, India.
}

\section{Supporting Information}

Figure S1. ESP mapped isodensity surface (0.001 a.u) in isolated (a) [Bmim][Trp] (b) [Bmim][Tyr] (c) [MBmim][Trp] (d) [MBmim][Tyr] ion pairs.

Figure S2. Color-filled RDG isosurfaces depicting Non-covalent interaction (NCI) regions in (a) [Bmim][Trp] and (b) [Bmim][Tyr], (c) [MBmim][Trp] and (d) [MBmim][Tyr] ion pairs. Green regions denote $0---H$ and $l p---\pi$ interactions while the red isosurface refers to steric effects. The NCI index plot of function 1 (sign $(\lambda 2) \rho$ values) on the $\mathrm{x}$ axis versus function 2, the reduced density gradient (RDG) on the Y-axis for the same have also been shown along with.

Table S1. Net NBO atomic charges in a.u for [Bmim][AA] and [MBmim][AA] ion-pairs.

Table S2. $\quad{ }^{17} \mathrm{O}$ and ${ }^{15} \mathrm{~N}$ NMR in $[\mathrm{Bmim}][\mathrm{AA}]$ and $[\mathrm{MBmim}][\mathrm{AA}]$ ion pairs.

Table S3. Spin-spin coupling constants for $J(\mathrm{O}, \mathrm{H})$ for inter- as well as intramolecular hydrogen bonding in $[\mathrm{Bmim}][\mathrm{AA}]$ and $[\mathrm{MBmim}][\mathrm{AA}]$ ion pairs (AA=Trp, Tyr) . See text for details.

Table S4. A comparison of selected vibrational frequencies ( $v=$ Stretching and $\delta=$ Bending) in [Bmim][AA] and [MBmim][AA] complexes. See text for details.

Figure S3. Infra red spectra for the (a) [Bmim][Trp], (b) [Bmim][Tyr], (c) [MBmim[Trp] and (d) $[\mathrm{MBmim}][\mathrm{Tyr}]$ ion-pairs. 


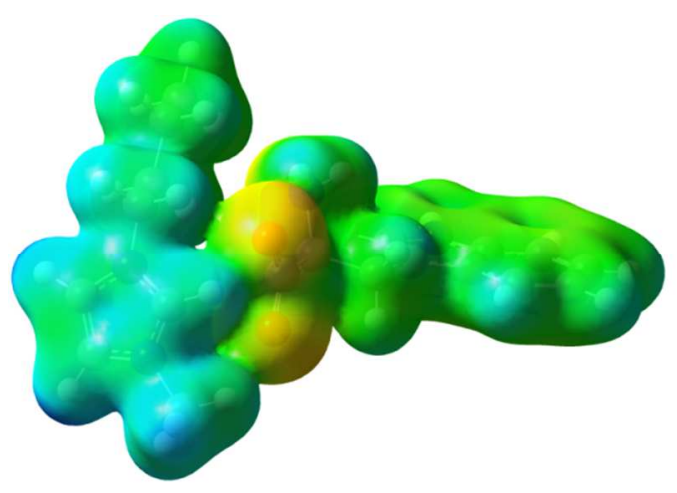

(a)

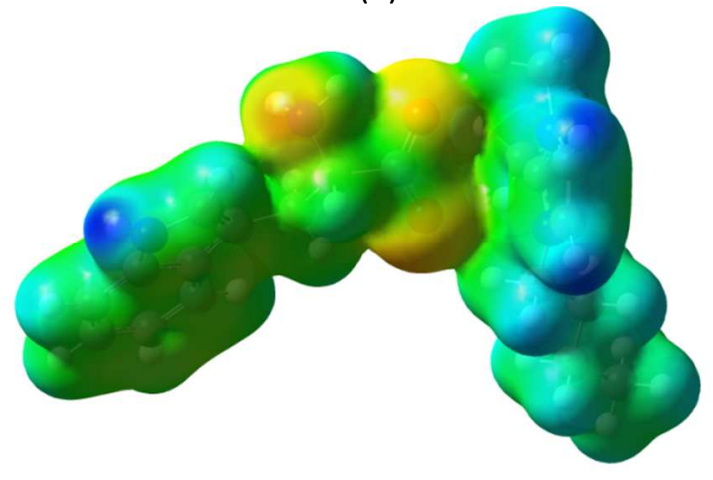

(c)

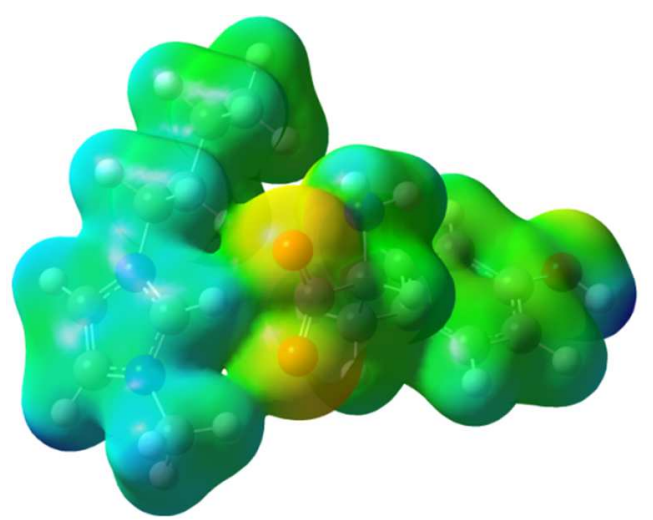

(b)

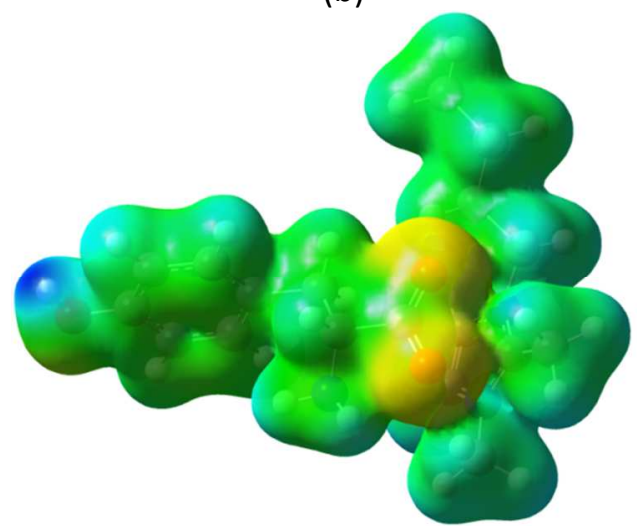

(d)

Figure S1. 
(a)
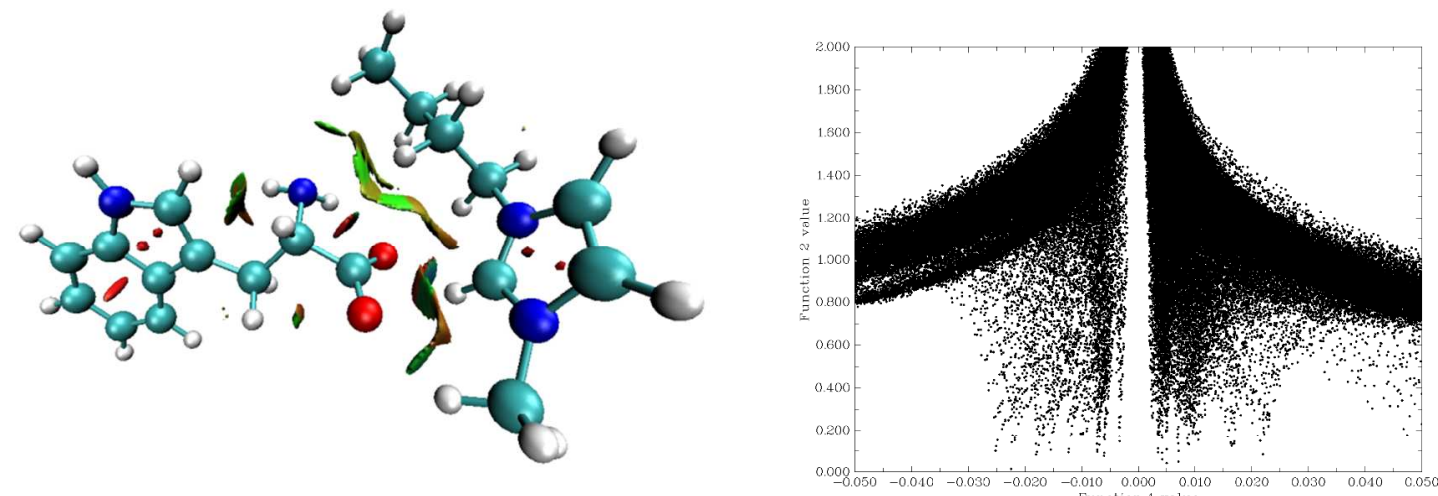

(b)
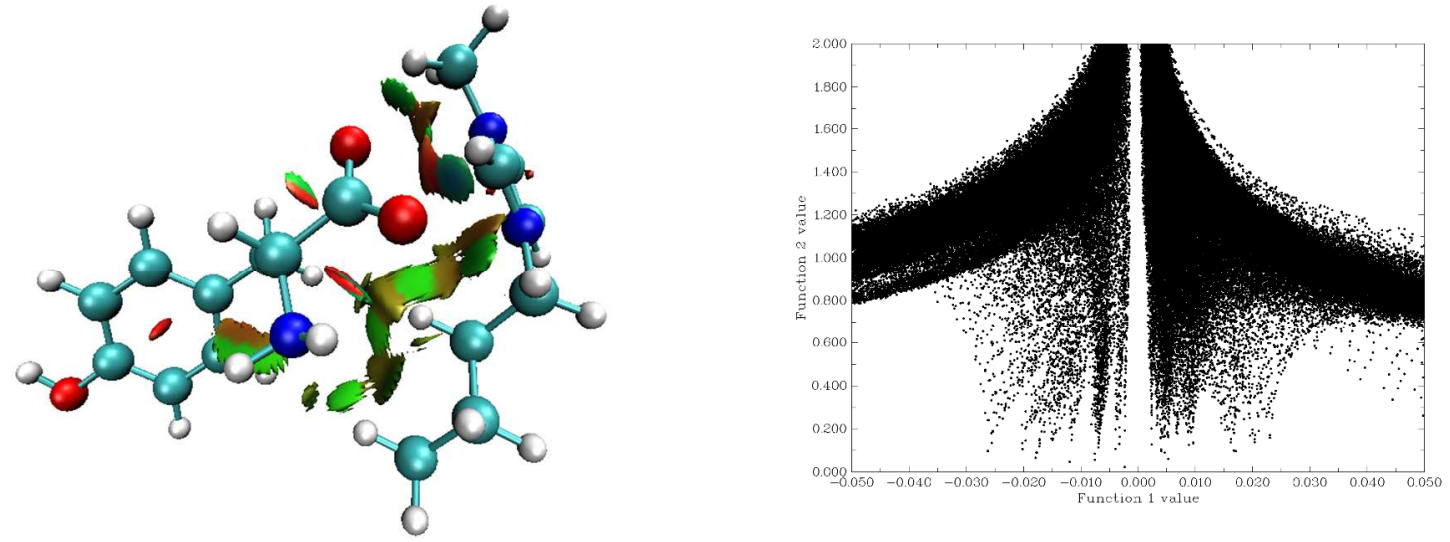

(c)

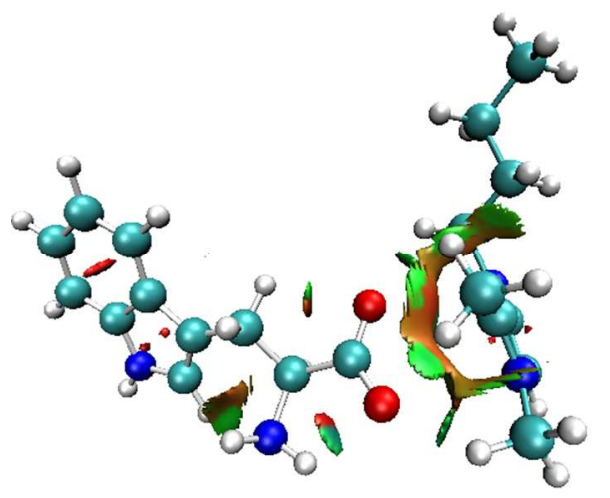

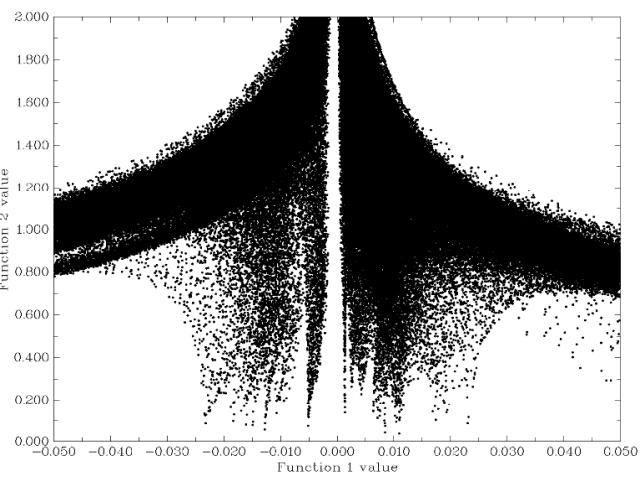

(d)
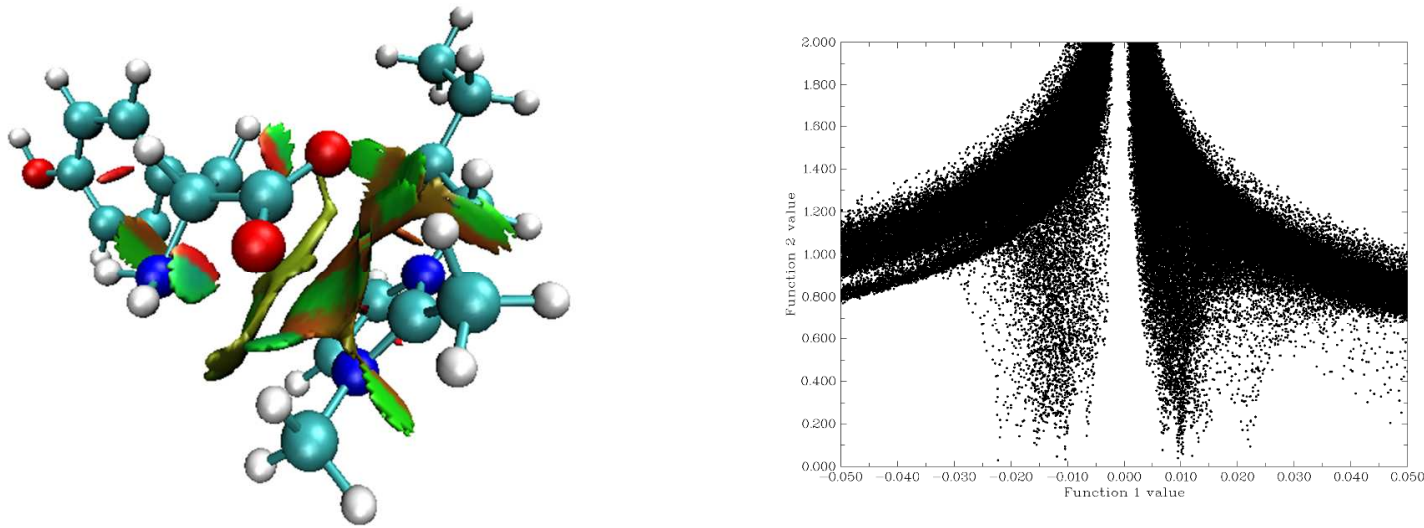

Figure S2. 
Table S1: Net NBO atomic charges in a.u for [Bmim][AA] and [MBmim][AA] ion-pairs.

\begin{tabular}{ccccccc}
\hline Atoms & {$[$ Bmim][Phe $]$} & {$[$ Bmim][Trp] } & {$[$ Bmim][Tyr] } & {$[\mathrm{MBmim}][\mathrm{Phe}]$} & {$[\mathrm{MBmim}][\mathrm{Trp}]$} & {$[\mathrm{MBmim}][\mathrm{Tyr}]$} \\
\hline $\mathrm{C} 1$ & 0.3202 & 0.3214 & 0.3199 & 0.5717 & 0.5607 & 0.5418 \\
$\mathrm{~N} 1$ & -0.3648 & -0.3643 & -0.3627 & -0.3750 & -0.3739 & -0.3716 \\
$\mathrm{~N} 2$ & -0.3657 & -0.3664 & -0.3668 & -0.3836 & -0.3722 & -0.3675 \\
N1' & -0.8778 & -0.8911 & -0.8883 & -0.8657 & -0.8813 & -0.8863 \\
N2' & & -0.5589 & & & -0.5596 & \\
O1 & -0.8302 & -0.8294 & -0.8260 & -0.7998 & -0.8419 & -0.8266 \\
O2 & -0.8170 & -0.8171 & -0.6913 & -0.8362 & -0.8346 & -0.8179 \\
$\mathrm{Ha}$ & 0.3578 & 0.3846 & 0.3861 & 0.3458 & 0.3856 & 0.3802 \\
$\mathrm{Hb}$ & 0.3754 & 0.3560 & 0.3585 & 0.3801 & 0.3467 & 0.3562 \\
\hline
\end{tabular}


Table S2: ${ }^{17} \mathrm{O}$ and ${ }^{15} \mathrm{~N}$ NMR in $[\mathrm{Bmim}][\mathrm{AA}]$ and $[\mathrm{MBmim}][\mathrm{AA}]$ ion pairs.

\begin{tabular}{|c|c|c|c|c|c|c|c|c|c|c|c|}
\hline$\delta_{0}$ & Bmim & MBmim & Phe & Trp & Tyr & [Bmim][Phe] & [Bmim][Trp] & [Bmim][Tyr] & [MBmim][Phe] & [MBmim][Trp] & [MBmim][Tyr] \\
\hline 01 & & & 355.2 & 351.9 & 356.3 & 369.2 & 373.8 & 377.2 & 397.9 & 360.0 & 370.4 \\
\hline 02 & & & 351.4 & 352.6 & 356.6 & 383.0 & 380.1 & 374.7 & 365.3 & 357.0 & 370.9 \\
\hline \multicolumn{12}{|l|}{$\delta_{\mathrm{N}}$} \\
\hline $\mathrm{N} 1$ & $\begin{array}{c}- \\
233.3\end{array}$ & -237.0 & & & & -240.8 & -240.4 & -240.5 & -239.2 & -236.6 & -238.2 \\
\hline $\mathrm{N} 2$ & $\begin{array}{c}- \\
212.0\end{array}$ & -219.6 & & & & -220.3 & -220.9 & -220.6 & -234.8 & -220.9 & -220.8 \\
\hline
\end{tabular}


Table S3: Spin-spin coupling constants for $J(0, \mathrm{H})$ for inter- as well as intramolecular hydrogen bonding in $[\mathrm{Bmim}][\mathrm{AA}]$ and $[\mathrm{MBmim}][\mathrm{AA}]$ ion pairs $(\mathrm{AA}=\mathrm{Trp}$, Tyr) . See text for details.

\begin{tabular}{cccccccc}
\hline & Inter & distances & FC & SD & PSO & DSO & total \\
\hline & O1---H1 & 2.292 & -0.674 & 0.037 & 0.501 & -0.374 & -0.510 \\
O2---H1 & 2.013 & 2.376 & -0.045 & 0.812 & -0.541 & 2.602 \\
a) $11---H 6$ & 2.208 & 2.555 & -0.091 & 0.657 & -0.487 & 2.634 \\
O2---H7 & 2.391 & 0.661 & 0.077 & 0.374 & -0.455 & 0.658 \\
N1'---H9 & 2.802 & -0.104 & -0.007 & -0.154 & -0.188 & -0.078 \\
O2---Ha & 2.116 & 1.172 & 0.009 & 0.682 & -0.472 & 2.181 \\
\hline
\end{tabular}

\begin{tabular}{lllllll}
\hline \multicolumn{1}{c}{ Inter } & & FC & SD & PSO & DSO & total \\
\hline O1---H1 & 2.29071 & -0.762 & 0.039 & 0.497 & -0.376 & -0.602 \\
O2---H1 & 1.99034 & 2.630 & 0.052 & 0.847 & -0.550 & 2.873 \\
b) $01---H 6$ & 2.40866 & 0.644 & 0.082 & 0.360 & -0.449 & 0.637 \\
O2---H7 & 2.22388 & 2.292 & -0.093 & 0.651 & -0.482 & 2.367 \\
N1'---H9 & 2.77131 & -0.161 & 0.003 & -0.169 & 0.198 & -0.129 \\
O2---Ha & 2.19410 & 1.333 & 0.088 & 0.611 & -0.418 & 1.614 \\
\hline
\end{tabular}

\begin{tabular}{|c|c|c|c|c|c|c|c|}
\hline & Inter & & FC & SD & PSO & DSO & total \\
\hline \multirow{6}{*}{ c) } & 01---H6 & 2.34000 & 0.724 & -0.005 & 0.432 & -0.423 & 0.718 \\
\hline & 01---H26 & 2.11495 & 3.290 & -0.087 & 0.759 & -0.879 & 3.429 \\
\hline & O2---H26 & 2.21743 & 2.101 & -0.064 & 0.702 & -0.539 & 2.200 \\
\hline & O2---H9 & 2.66702 & -0.728 & 0.005 & 0.261 & -0.306 & -0.768 \\
\hline & O2---H7 & 2.13245 & 1.861 & 0.096 & 0.661 & -0.438 & 2.179 \\
\hline & 01---H11 & 2.45935 & -0.070 & 0.08 & 0.434 & -0.320 & 0.122 \\
\hline
\end{tabular}

\begin{tabular}{lllllll}
\hline \multicolumn{1}{c}{ Inter } & & FC & SD & PSO & DSO & total \\
\hline 01---H6 & 2.45371 & -0.402 & -0.007 & 0.414 & -0.350 & -0.344 \\
O1---H26 & 2.49720 & 0.219 & 0.007 & 0.380 & -0.374 & 0.232 \\
d) $02---H 26$ & 2.16140 & 2.686 & 0.031 & 0.447 & -0.526 & 2.735 \\
O2---H9 & 2.46094 & 0.236 & 0.127 & 0.389 & -0.438 & 0.199 \\
O2---H7 & 2.47300 & 0.250 & 0.024 & 0.459 & -0.459 & 0.275 \\
O1---H11 & 2.50443 & -0.239 & 0.042 & 0.452 & -0.314 & -0.059 \\
\hline
\end{tabular}


Table S4: A comparison of selected vibrational frequencies ( $v=$ Stretching and $\delta=$ Bending) in [Bmim][AA] and [MBmim][AA] complexes. See text for details.

\begin{tabular}{|c|c|c|c|c|c|c|c|}
\hline Assignments & [Bmim] & [Phe] & [Trp] & [Tyr] & {$[\mathrm{Bmim}][\mathrm{Phe}]$} & {$[\mathrm{Bmim}][\mathrm{Trp}]$} & {$[\mathrm{Bmim}][\mathrm{Tyr}]$} \\
\hline$v \mathrm{O}-\mathrm{H}$ & & & & 3922 & & & 3918 \\
\hline$v \mathrm{~N}-\mathrm{H}$ & & & 3758 & & & 3744 & \\
\hline$v \mathrm{NH}_{2}$ & & 3574,3492 & 3571,3475 & 3642,3481 & 3622,3538 & 3617,3532 & 3649,3565 \\
\hline $\begin{array}{c}v \mathrm{C} 2 \mathrm{H} 2+ \\
v \mathrm{C} 3 \mathrm{H} 3\end{array}$ & 3324,3306 & & & & 3353,3335 & & 3336,3317 \\
\hline$v \mathrm{C}-\mathrm{H}_{\text {pyrol }}$ & & & 3289 & & & 3302 & \\
\hline$v \mathrm{C}-\mathrm{H}_{\text {phe }}$ & & $3238,3229,3217$ & 3535,3225 & 3237,3220 & 3253,3240 & 3255,3244 & 3246,3219 \\
\hline$v \mathrm{C} 1-\mathrm{H} 1$ & 3305 & & & & 3239 & 3244 & 3233 \\
\hline$v \mathrm{CH}_{3}{ }^{\mathrm{a}}$ & $3210,3202,3115$ & & & & $3209,3197,3111$ & & $3196,3175,3089$ \\
\hline$v \mathrm{CH}_{2}^{\mathrm{a}}$ & 3167,3114 & & & & $3209,3197,3111$ & & 3183,3113 \\
\hline$v \mathrm{C}-\mathrm{N}$ & 1647 & & & & 1628 & 1630 & 1629 \\
\hline$v \mathrm{C}-\mathrm{C}_{\text {phe }}$ & & 1694 & & 1708 & 1393 & 1706 & 1714 \\
\hline$v \mathrm{C}=0$ & & 1713 & 1715 & 1722 & 1677 & 1681 & 1674 \\
\hline$\delta \mathrm{NH}_{2}$ & & & 1678 & 1633 & 1616 & 1621 & \\
\hline Assignments & [MBmim] & [Phe] & [Trp] & [Tyr] & [MBmim][Phe] & [MBmim][Trp] & [MBmim][Tyr] \\
\hline$v \mathrm{O}-\mathrm{H}$ & & & & 3922 & & & 3920 \\
\hline$v \mathrm{~N}-\mathrm{H}$ & & & 3758 & & & 3764 & \\
\hline$v \mathrm{NH}_{2}$ & & 3574,3492 & 3571,3475 & 3642,3481 & 3611,3545 & 3612,3539 & 3632,3554 \\
\hline $\begin{array}{c}v \mathrm{C} 2 \mathrm{H} 2+ \\
v \mathrm{C} 3 \mathrm{H} 3\end{array}$ & 3353,3331 & & & & 3340,3320 & 3346,3327 & 3353,3332 \\
\hline$v \mathrm{C}-\mathrm{H}_{\text {pyrol }}$ & & & 3289 & & & 3291 & \\
\hline$v \mathrm{C}-\mathrm{H}_{\mathrm{phe}}$ & & $3238,3229,3217$ & 3535,3225 & 3237,3220 & 3241,3231 & $3239,3225,3214$ & 3243,3218 \\
\hline$v \mathrm{CH}_{3}{ }^{\mathrm{b}}$ & $3198,3150,3089$ & & & & $3184,3142,3071$ & $3205,3161,3079$ & 3154,3082 \\
\hline$v \mathrm{CH}_{3}{ }^{\mathrm{a}}$ & $3204,3202,3114$ & & & & $3196,3150,3051$ & $3201,3187,3101$ & 3188,3101 \\
\hline$v \mathrm{CH}_{2} \mathrm{a}^{\mathrm{a}}$ & 3168,3132 & & & & 3123,3069 & 3172,3123 & 3146 \\
\hline$v \mathrm{C}-\mathrm{N}$ & 1608 & & & & 1615 & 1608 & 1608 \\
\hline$v \mathrm{C}-\mathrm{C}_{\mathrm{phe}}$ & & 1694 & & 1708 & 1700 & 1707 & 1710 \\
\hline$v \mathrm{C}=0$ & & 1713 & 1715 & 1722 & 1658 & 1657 & 1680 \\
\hline$\delta \mathrm{NH}_{2}$ & & & 1678 & 1633 & 1681 & 1683 & 1621 \\
\hline
\end{tabular}



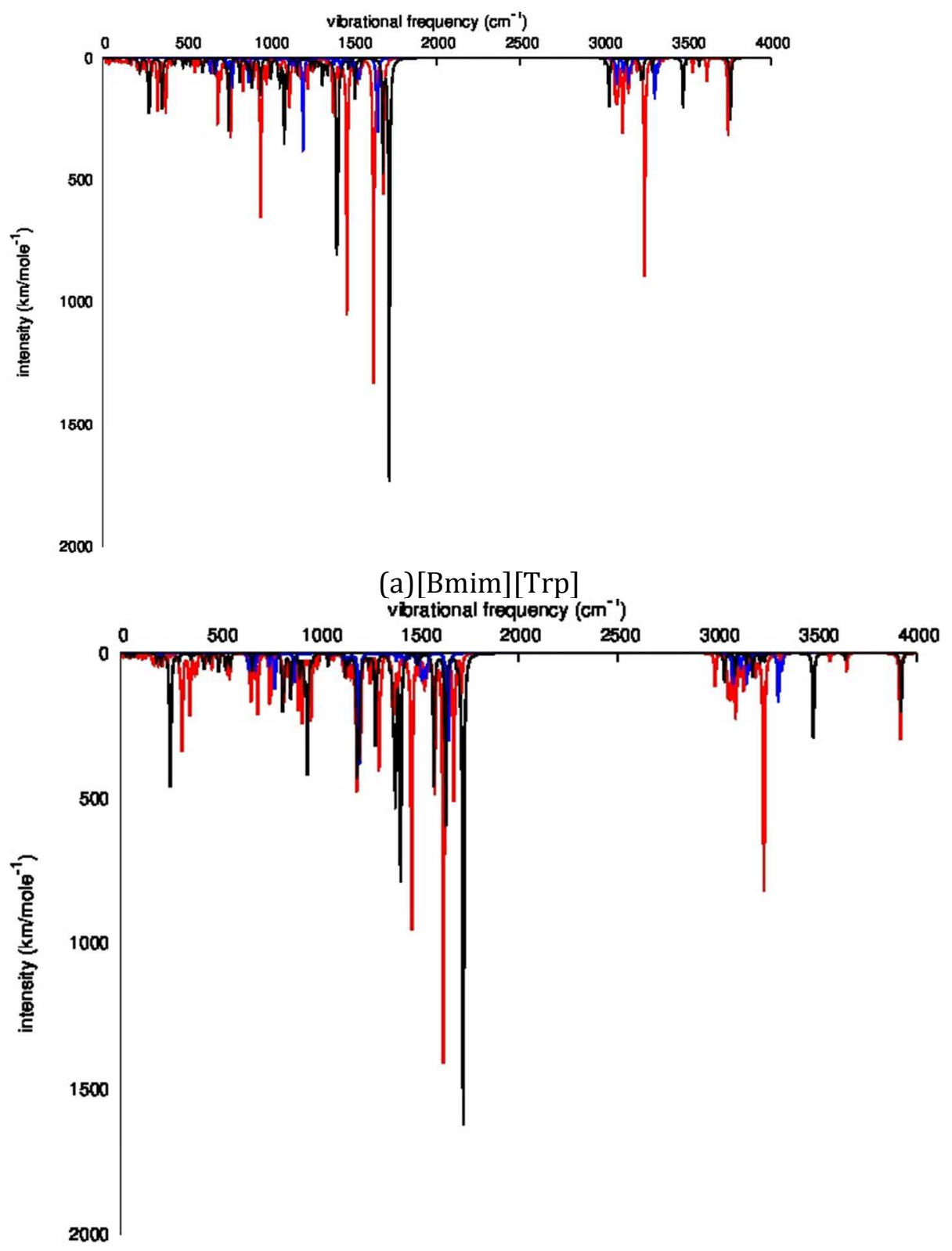

(b) $[$ Bmim] $]$ Tyr]

Figure S3 cont. 

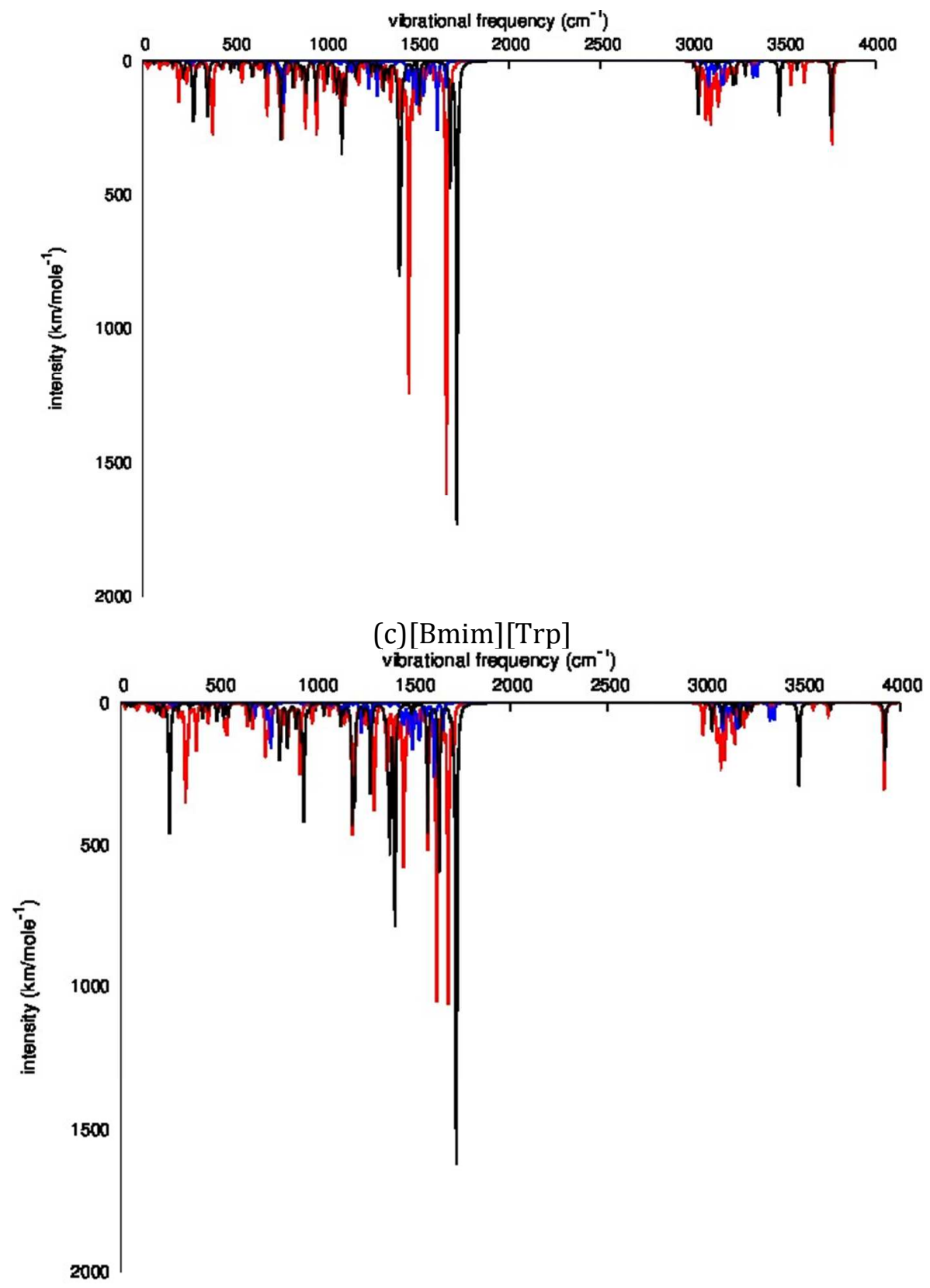

(d) [Bmim][Tyr]

Figure S3. 